\title{
Software Reliability Data Analysis with Marshall-Olkin Extended Weibull Model using MCMC Method for Non- Informative Set of Priors
}

\author{
Ashwini K. Srivastava \\ Department of Computer Application, \\ S.Kisan P.G. College, Basti, \\ U.P., India
}

\author{
Vijay Kumar \\ Department of Mathematics and Statistics, \\ D.D.U. Gorakhpur University, Gorakhpur, \\ U.P., India
}

\begin{abstract}
In this paper, the two-parameter Marshall-Olkin Extended Weibull (MOEW) model is considered to analyze the software reliability data. The Markov Chain Monte Carlo (MCMC) method is used to compute the Bayes estimates of the model parameters. In this paper, it is assumed that the parameters have non-informative set of priors and they are independently distributed. Under the above priors, we use Gibbs algorithm in OpenBUGS to generate MCMC samples from the posterior density function. Based on the generated samples, we can compute the Bayes estimates of the unknown parameters and also can construct highest posterior density credible intervals. We also compute the maximum likelihood estimate and associated confidence intervals to compare the performances of the Bayes estimators with the classical estimators. One data analysis is performed for illustrative purposes.
\end{abstract}

\section{Keywords}

Marshall-Olkin Extended Weibull (MOEW) model, Parameter estimation, Maximum likelihood estimate (MLE), Bayes estimates, Markov Chain Monte Carlo (MCMC).

\section{INTRODUCTION}

The exponentiated Weibull family model has been used to analyze the data of which the hazard-rate function can be increasing, decreasing, bathtub-shaped, or unimodal [13], [14], [21], [24] and [25]. The Exponentiated Weibull model is considered as the intensity function of nonhomogeneous Poisson process in software reliability analysis in [3]. Detailed reviews on the Exponentiated Weibull with various new statistical measures are given in [15] and [22]. Jiang and Murthy [7] discussed the parameter estimation of the model by graphical approaches.

This article addresses the extended Weibull distribution in [11]. The purpose is to investigate some properties of this model, parameter estimation using different approaches, and to study the versatility of the model in modeling software reliability data. This model is constructed by a general method of introducing a parameter to a model such that if $\overline{\mathrm{F}}(\mathrm{x})=1-\mathrm{F}(\mathrm{x})$ is a survival function with one parameter $\alpha$, a two-parameter family of survival functions is generated as

$$
\begin{aligned}
& \overline{\mathrm{G}} \mathrm{x} ; \alpha, \lambda=\frac{\lambda \overline{\mathrm{F}}(\mathrm{x})}{1-(1-\lambda) \overline{\mathrm{F}}(\mathrm{x})} ; \quad-\infty<\mathrm{x}<\infty, \alpha>0, \lambda>0 . \\
& \text { If } . \lambda=1 \text { then } \overline{\mathrm{G}} \mathrm{x}=\overline{\mathrm{F}} \mathrm{x}
\end{aligned}
$$

\subsection{Model Analysis}

The extended Weibull model is constructed by a general method of introducing a parameter to expand the family of Weibull models [11] and [12]. From equation (1.1), the survival function is

$$
\mathrm{S}(\mathrm{x} ; \alpha, \lambda)=\frac{\lambda \exp -\mathrm{x}^{\alpha}}{1-(1-\lambda) \exp -\mathrm{x}^{\alpha}} \quad ;(\alpha, \lambda)>0, \mathrm{x}>0
$$

The $\mathrm{R}$ function sext.weibull() given in reliaR package[8] computes the reliability/ survival function.

The cumulative distribution function (cdf) can be written as

$$
\mathrm{F}(\mathrm{x} ; \alpha, \lambda)=\frac{1-\exp -\mathrm{x}^{\alpha}}{1-(1-\lambda) \exp -\mathrm{x}^{\alpha}} \quad ;(\alpha, \lambda)>0, \mathrm{x}>0
$$

Here $\alpha$ and $\lambda$ are the shape and tilt parameters, respectively. The probability density function (pdf) associated with (1.3) is given by

$$
\mathrm{f} x ; \alpha, \lambda=\frac{\lambda \alpha \mathrm{x}^{\alpha-1} \exp -\mathrm{x}^{\alpha}}{1-(1-\lambda) \exp -\mathrm{x}^{\alpha}} ;(\mathrm{x}>0, \lambda>0, \alpha>0)
$$

We shall write $\operatorname{MOEW}(\alpha, \lambda)$ to denote Marshall-Olkin Extended Weibull (MOEW) model with parameters $\alpha$ and $\lambda$. The $\mathrm{R}$ functions dext.weibull() and pext.weibull() given in [8] can be used for the computation of pdf and cdf, respectively. 


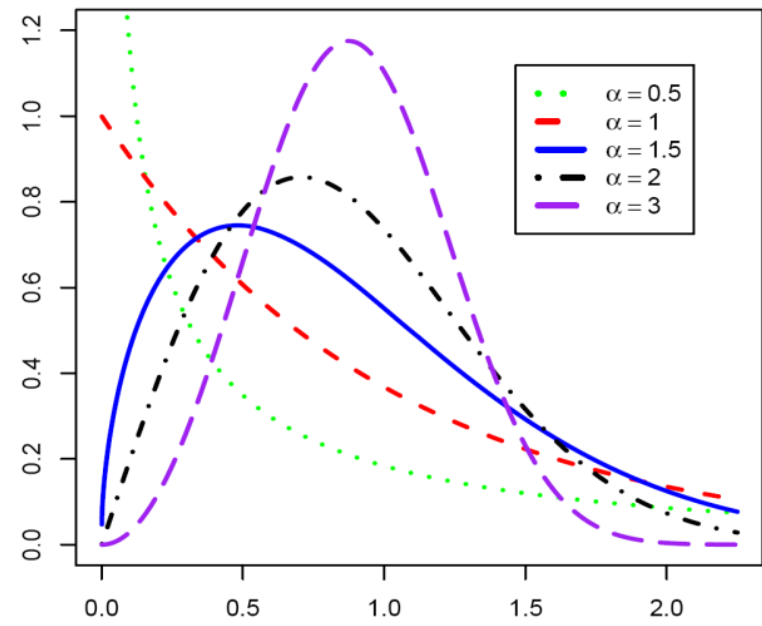

Fig 1: Plots of the probability density function of the MOEW Model for $\lambda=1$ and different values of $\alpha$

\section{Characterization of pdf}

Case $1: \lambda \leq 1$

(i) $\alpha \leq 1, f(x)$ is decreasing in $x$

(ii) $\alpha>1, f(x)$ is unimodal,

Case 2: $\lambda>1$

(i) $\quad \alpha>1, f(x)$ is unimodal

(ii) $\quad \alpha<1$, when $\alpha$ is quite small, $\mathrm{f}(\mathrm{x})$ is decreasing in $\mathrm{x}$, whereas $\mathrm{f}(\mathrm{x})$ is initially decreasing quickly, then increasing and eventually decreasing when $\lambda$ is large enough.

Some of the typical MOEW density functions for different values of $\alpha$ and for $\lambda=1$ are depicted in Figure1. It is clear from the Figure 1 that the density function of the MOEW model can take different shapes.

\subsubsection{The quantile function}

For a continuous distribution $\mathrm{F}(\mathrm{x})$, the $\mathrm{p}$ percentile (also referred to as fractile or quantile), $x_{p}$, for a given $p, 0<p<1$, is a number such that

$$
\mathrm{P}\left(\mathrm{X} \leq \mathrm{x}_{\mathrm{p}}\right)=\mathrm{F}\left(\mathrm{x}_{\mathrm{p}}\right)=\mathrm{p}
$$

The quantile function of Marshall-Olkin Extended Weibull (MOEW) model can be obtained by solving

$$
\mathrm{x}_{\mathrm{p}}=\left\{\log \left(1+\frac{\lambda \mathrm{p}}{(1-\mathrm{p})}\right)\right\}^{1 / \alpha} \text {. }
$$

The computation of quantiles the $\mathrm{R}$ function qext.weibull( ), given in [8], can be used. In particular, for $p=0.5$ we get

$$
\operatorname{Median}\left(\mathrm{x}_{0.5}\right)=\log 1+\lambda^{1 / \alpha}
$$

\subsubsection{The random deviate generation}

The random deviate can be generated from $\operatorname{MOEW}(\alpha, \lambda)$ by

$$
\mathrm{x}=\left\{\log \left(1+\frac{\lambda \mathrm{u}}{(1-\mathrm{u})}\right)\right\}^{1 / \alpha} ; 0<\mathrm{u}<1
$$

where $\mathrm{u}$ has the $\mathrm{U}(0,1)$ distribution. The $\mathrm{R}$ function rext.weibull(), given in [8], generates the random deviate from $\operatorname{MOEW}(\alpha, \lambda)$.

\subsubsection{The Hazard Function(hrf)}

The Hazard Function of Marshall-Olkin Extended Weibull (MOEW) model is:

$$
\mathrm{h} x ; \alpha, \lambda=\frac{\alpha \mathrm{x}^{\alpha-1}}{1+(1-\lambda) \exp -\mathrm{x}^{\alpha}},(\alpha, \lambda)>0 \text { and } \mathrm{x} \geq 0
$$

and the allied $\mathrm{R}$ function hext.weibull( ) given in [8]

Some of the typical Marshall-Olkin Extended Weibull (MOEW) model hazard functions for different values of $\alpha$ and for $\lambda=1$ are depicted in Figure2. It is clear from the Figure 2 that the hazard function of the Marshall-Olkin Extended Weibull (MOEW) model can take different shapes

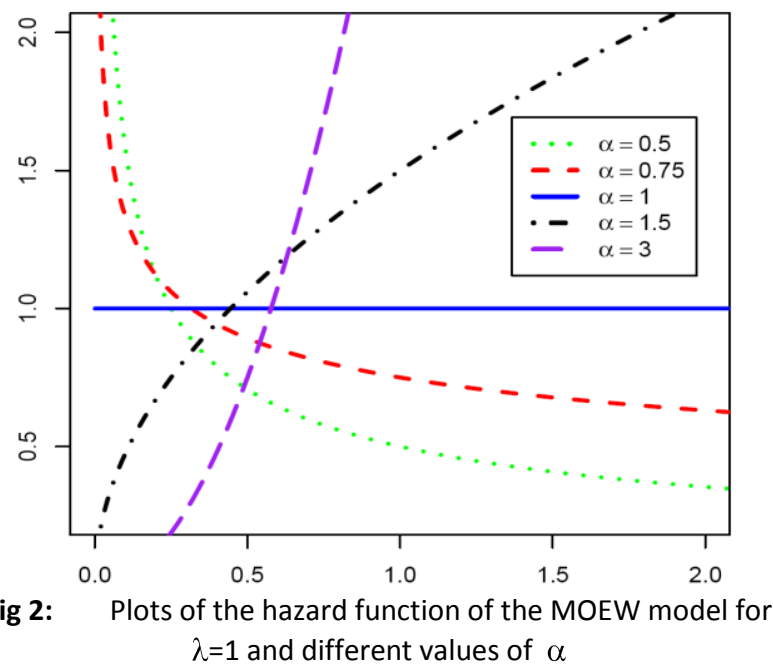

\section{Characterization of hrf}

In summary, the characterization of $h r f$ is as follows [11]:

(i) $\lambda \geq 1$ and $\alpha>1$, or $\lambda>1$ and $\alpha \geq 1$, then $\mathrm{h}(\mathrm{x})$ is increasing.

(ii) $\lambda \leq 1$ and $\alpha<1$, or $\lambda<1$ and $\alpha \leq 1$, then $\mathrm{h}(\mathrm{x})$ is decreasing.

(iii) $\alpha>1$ and $\lambda<1$, then $\mathrm{h}(\mathrm{x})$ is initially increasing and eventually increasing, but there may be an interval where it is decreasing. When $\lambda$ is small enough, the curve of $h(x)$ has local maximum extreme value point $\left(\mathrm{x}=\mathrm{x}_{1}{ }^{*}\right)$, and after the point, it takes bathtub-shaped. This implies that the curve of $\mathrm{h}(\mathrm{x})$ has a local minimum extreme value point, i.e., the change point $\left(\mathrm{x}=\mathrm{x}_{2}{ }^{*}\right)$ which is obtained by solving

$$
\begin{aligned}
& 1-1-\lambda \exp -\mathrm{x}^{\alpha}- \\
& \alpha 1-\lambda / \alpha-1 \quad \mathrm{x}^{\alpha} \cdot \exp -\mathrm{x}^{\alpha}=0
\end{aligned}
$$


(iv) Above Eq. (1.10) is obtained from Eq. (1.9) by taking the first derivative with respect to $\mathrm{x}$. It has two possible solutions; the smaller one is $\mathrm{x}_{1}{ }^{*}$ and the larger is $\mathrm{x}_{2}{ }^{*}$.

(v) $\quad \alpha<1$ and $\lambda>1$, then $\mathrm{h}(\mathrm{x})$ is initially decreasing and eventually decreasing, but there may be an interval where it is increasing. In the case, when $\lambda$ is large enough, there are two local extreme value points: one is at $\mathrm{x}=\mathrm{x}_{1}{ }^{*}$ and the other at $\mathrm{x}=\mathrm{x}_{2}{ }^{*}$, which are obtained from Eq. (1.10). $\mathrm{x}_{1}{ }^{*}$ and $\mathrm{x}_{2}{ }^{*}$ correspond to the smaller and larger solutions, respectively.

\subsubsection{The cumulative hazard function}

The cumulative hazard function $\mathrm{H}(\mathrm{x})$ defined as

$$
\mathrm{H}(\mathrm{x})=-1-\log \mathrm{F}(\mathrm{x})
$$

can be obtained with the help of pext.weibull( ) function given in [8] by choosing arguments lower.tail $=F A L S E$ and log.p=TRUE. i.e.

- pext.weibull(x,alpha,lambda, lower.tail=FALSE, log.p = TRUE)

\subsubsection{Failure rate average (fra) and Conditional survival function(crf)}

Two other relevant functions useful in reliability analysis are failure rate average (fra) and conditional survival function (crf). The failure rate average of $\mathrm{X}$ is given by

$$
\operatorname{FRA}(\mathrm{x})=\frac{\mathrm{H}(\mathrm{x})}{\mathrm{x}} \quad, \mathrm{x}>0,
$$

where $H(x)$ is the cumulative hazard function. The conditional survival of $\mathrm{X}$ is defined by

$$
S(x \mid t)=\frac{S(x+t)}{S(x)}, t>0, x>0, S(\cdot)>0,
$$

respectively, where $F(\cdot)$ is the cdf of $X$. Similarly to $h(x)$ and FRA(x), the distribution of $\mathrm{X}$ belongs to the new better than used (NBU), exponential, or new worse than used (NWU) classes, when $\mathrm{S}(\mathrm{x} \mid \mathrm{t})<\mathrm{S}(\mathrm{x}), \mathrm{S}(\mathrm{t} \mid \mathrm{x})=\mathrm{S}(\mathrm{x})$, or $\mathrm{S}(\mathrm{x} \mid \mathrm{t})>\mathrm{S}(\mathrm{x})$, respectively.

The R functions hra.ext.weibull( ) and crf.ext.weibull( ) given in [8] can be used for the failure rate average (fra) and conditional survival function(crf), respectively.

\section{MAXIMUM ESTIMATION}

\section{LIKELIHOOD}

Let $\underline{x}=\left(x_{1}, \ldots, x_{n}\right)$ be a sample of size $n$ from $\operatorname{MOEW}(\alpha, \lambda)$, then the $\log$-likelihood function $\mathrm{L}(\alpha, \lambda)$ can be written as[23];

$$
\begin{aligned}
\log L= & n \log \alpha+n \log \lambda+\alpha-1 \sum_{i=1}^{n} \log x_{i}-\sum_{i=1}^{n} x_{i}^{\alpha} \\
& -2 \sum_{i=1}^{n} \log 1-(1-\lambda) \exp -x_{i}^{\alpha}
\end{aligned}
$$

Therefore, to obtain the MLE's of $\alpha$ and $\lambda$ we can maximize (2.1) directly with respect to $\alpha$ and $\lambda$ or we can solve the following two non-linear equations using iterative procedure:

$$
\begin{aligned}
\frac{\partial \log L}{\partial \alpha} & =\frac{n}{\alpha}+\sum_{i=1}^{n} \log x_{i} \\
& -\sum_{i=1}^{n} \frac{x_{i}^{\alpha} \log x_{i} 1+(1-\lambda) \exp -x_{i}^{\alpha}}{1-(1-\lambda) \exp -x_{i}^{\alpha}}=0
\end{aligned}
$$

$$
\frac{\partial \log \mathrm{L}}{\partial \lambda}=\frac{\mathrm{n}}{\lambda}-2 \sum_{\mathrm{i}=1}^{\mathrm{n}} \frac{1}{\exp -\mathrm{x}_{\mathrm{i}}^{\alpha}-(1-\lambda)}=0 .
$$

\subsection{Information Matrix and Asymptotic Confidence Intervals}

Since the MLEs of the unknown parameters $\theta=(\alpha, \lambda)$ can not be obtained in closed forms, it is not easy to derive the exact distributions of the MLEs. We can derive the asymptotic confidence intervals of these parameters when $\alpha>0$, and $\lambda>0$. The simplest large sample approach is to assume that the MLE $(\hat{\alpha}, \hat{\lambda})$ are approximately bivariate normal with mean $(\alpha, \lambda)$ and covariance matrix $\mathrm{I}_{0}^{-1}$ [9], where $\mathrm{I}_{0}^{-1}$ is the inverse of the observed information matrix

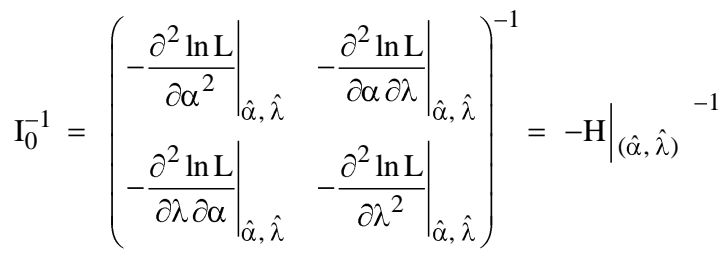

$$
\begin{aligned}
& =\left(\begin{array}{cc}
\operatorname{var}(\hat{\alpha}) & \operatorname{cov}(\hat{\alpha}, \hat{\lambda}) \\
\operatorname{cov}(\hat{\alpha}, \hat{\lambda}) & \operatorname{var}(\hat{\lambda})
\end{array}\right) .
\end{aligned}
$$

The above approach is used to derive the $100(1-\gamma / 2) \%$ confidence intervals of the parameters $\theta=(\alpha, \lambda)$ as in the following forms

$$
\hat{\alpha} \pm z_{\gamma / 2} \sqrt{\operatorname{Var}(\hat{\alpha})} \text { and } \hat{\lambda} \pm z_{\gamma / 2} \sqrt{\operatorname{Var}(\hat{\lambda})}
$$

Here, $Z_{\gamma / 2}$ is the upper $(\gamma / 2)$ th percentile of the standard normal distribution.

\subsection{Computation of Maximum Likelihood Estimation}

We are using software reliability data set SYS2.DAT - 86 timebetween-failures [10] is considered for illustration of the proposed methodology. In this real data set, Time-between-failures is converted to time to failures and scaled.

In MOEW model, the direct maximization of log-likelihood function given in (2.1) using Newton-Raphson method in R gives, the ML estimates and standard error. The $95 \%$ confidence interval is computed using (2.4) and (2.5). The Table 1 shows the ML estimates, standard error(SE) and $95 \%$ Confidence Intervals of the parameters alpha and lambda. 
Table 1 Maximum likelihood estimate, standard error and 95\% confidence interval

\begin{tabular}{|c|c|c|c|}
\hline Parameter & MLE & Std. Error & 95\% Confidence Interval \\
\hline alpha & 0.3035937 & 0.0049873 & $(0.29381859,0.31336880)$ \\
\hline lambda & 279.21777 & 2.969300 & $(273.397947,285.037603)$ \\
\hline
\end{tabular}

\section{MODEL VALIDATION}

To study the goodness of fit of the Marshall-Olkin Extended Weibull (MOEW) model, we compute the Kolmogorov-Smirnov statistic between the empirical distribution function and the fitted distribution function when the parameters are obtained by method of maximum likelihood. For this we can use $\mathrm{R}$ function ks.ext.weibull( ), given in [8]. The result of $\mathrm{K}-\mathrm{S}$ test is $\mathrm{D}=0.0708$ with the corresponding $\mathrm{p}$-value $=0.7545$, Therefore, the high $\mathrm{p}$ value clearly indicates that Marshall-Olkin Extended Weibull (MOEW) model can be used to analyze this data set, and we also plot the empirical distribution function and the fitted distribution function in Figure 3. From above result and Figure3, it is clear that the estimated Marshall-Olkin Extended Weibull (MOEW) model provides excellent fit to the given data.

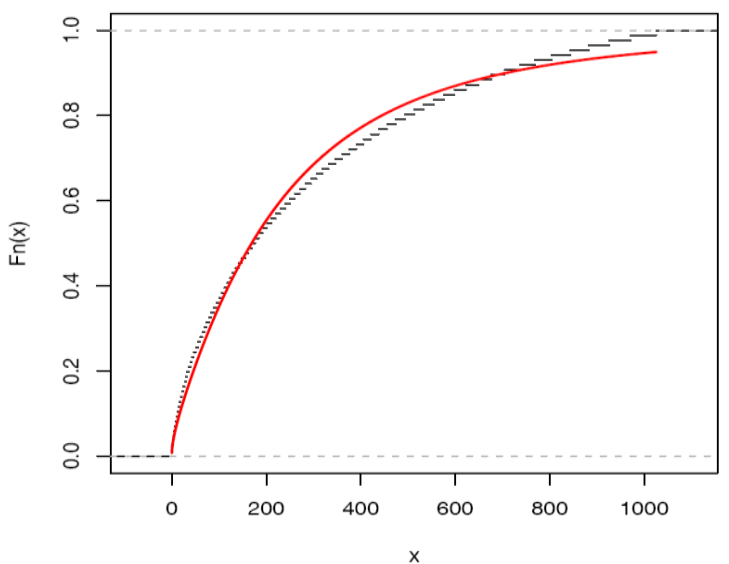

Fig 3: The graph for empirical distribution function and fitted distribution function.

There are two other graphical methods widely used i.e. QuantileQuantile (Q-Q) plots and the Probability-Probability (P-P) plots for checking whether a fitted model is in agreement with the data. In other words, we measure the quality of the fitted model. The pattern of points on a graph can be far more informative than the values of overall test statistics.

\subsection{The Q-Q Plots}

Let $\hat{\mathrm{F}}(\mathrm{x})$ be an estimate of $\mathrm{F}(\mathrm{x})$ based on $\mathrm{x}_{1}, \mathrm{x}_{2}, \ldots, \mathrm{x}_{\mathrm{n}}$. The scatter plot of the points

$$
\hat{\mathrm{F}}^{-1}\left(\mathrm{p}_{1: \mathrm{n}}\right) \text { versus } \mathrm{x}_{\mathrm{i}: \mathrm{n}}, \mathrm{i}=1,2, \ldots, \mathrm{n} \text {, is called a Q-Q plot. }
$$

Thus, the Q-Q plots show the estimated versus the observed quantiles. If the model fits the data well, the pattern of points on the Q-Q plot will exhibit a 45-degree straight line. Note that all the points of a Q-Q plot are inside the square

$$
\left[\hat{\mathrm{F}}^{-1}\left(\mathrm{p}_{1: \mathrm{n}}\right), \hat{\mathrm{F}}^{-1}\left(\mathrm{p}_{\mathrm{n}: \mathrm{n}}\right)\right] \times \mathrm{x}_{1: \mathrm{n}}, \mathrm{x}_{\mathrm{n}: \mathrm{n}}
$$

The corresponding $\mathrm{R}$ function qq.ext.weibull ( ) is given in [8]. As can be seen from the straight line pattern in Figure4, the MarshallOlkin Extended Weibull (MOEW) model fits the data very well.

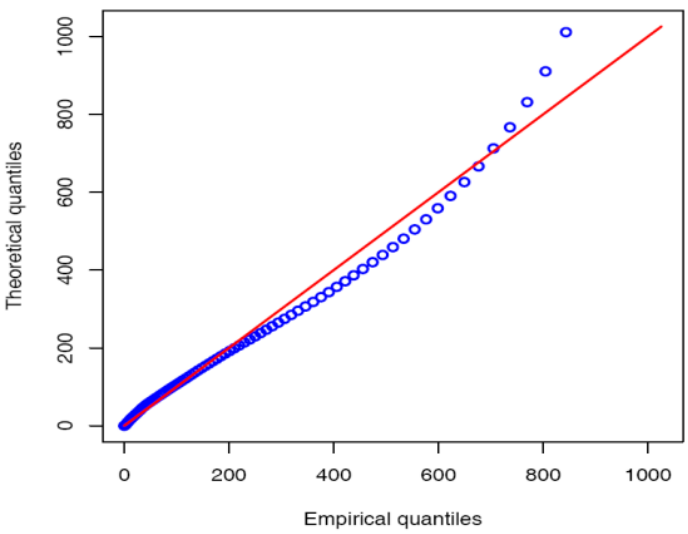

Fig 4: Quantile-Quantile(Q-Q) plot using MLEs as estimate.

\subsection{The P-P Plots}

Let $\mathrm{x}_{\mathrm{l}}, \mathrm{x}_{2}, \ldots, \mathrm{x}_{\mathrm{n}}$ be a sample from a given population with estimated cdf $\hat{F}(x)$. The scatter plot of the points $\hat{F}\left(x_{1: n}\right)$ versus $\mathrm{p}_{\mathrm{i}: \mathrm{n}}, \mathrm{i}=1,2, \ldots, \mathrm{n}$, is called a P-P plot. If the model fits the data well, the graph will be close to the 45-degree line. Here we note that all the points in the P-P plot are inside the unit square [0, 1] $\mathrm{x}[0,1]$. The corresponding $\mathrm{R}$ function pp.ext.weibull( $)$ is given in $[8]$.

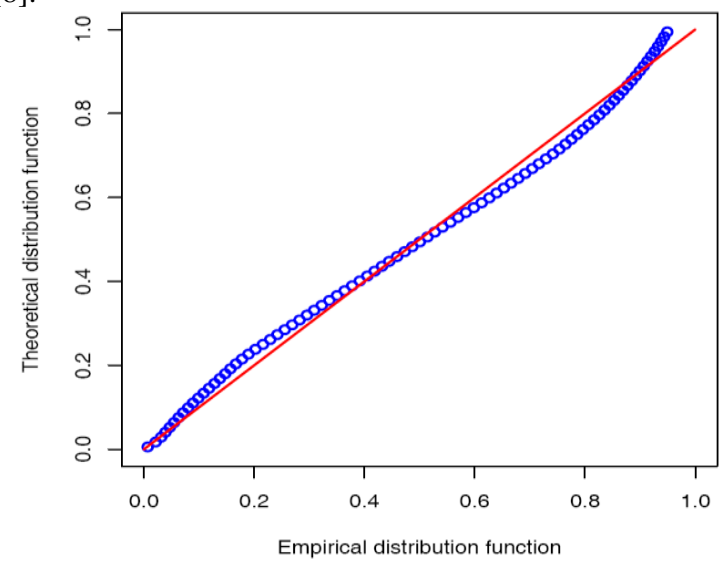

Fig 5: Probability-Probability(P-P) plot using MLEs as estimate.

As can be seen from the straight line pattern in Figure5 the Marshall-Olkin Extended Weibull (MOEW) model fits the data very well.

\section{BAYESIAN ESTIMATION USING MCMC TECHNIQUE}

The most widely used piece of software for applied Bayesian inference is the OpenBUGS. The software offers a user-interface, based on dialogue boxes and menu commands, through which the model may then be analyzed using Markov Chain Monte Carlo 
techniques. It is a fully extensible modular framework for constructing and analyzing Bayesian full probability models [18] for the existing probability models. As the MOEW model is not available in OpenBUGS. Thus it requires incorporation of a module to estimate parameters of MOEW model.

A module dext.weibull_T(alpha, lambda) is written in component Pascal, enables to perform full Bayesian analysis of Marshall-Olkin Extended Weibull(MOEW) model into OpenBUGS using the method described in [19] and [20].

\subsection{Implementation of Module - dext.weibull_T(alpha, lambda):}

The developed module is implemented to obtain the Bayes estimates of the MOEW model using MCMC method. The main function of the module is to generate MCMC sample from posterior distribution for non-informative set of priors, i.e. uniform priors.

\subsubsection{Uniform Prior distributions}

It frequently happens that the experimenter knows in advance that the probable values of $\theta$ lie over a finite range $[a, b]$ but has no strong opinion about any subset of values over this range. In such a case a uniform distribution over $[a, b]$ may be a good approximation of the prior distribution, its p.d.f. is given by

$$
\pi(\theta)= \begin{cases}\frac{1}{b-a} & ; 0<\mathbf{a}<\theta<\mathbf{b} \\ 0 & ; \text { otherwise }\end{cases}
$$

\subsection{OpenBUGS code to run MCMC)}

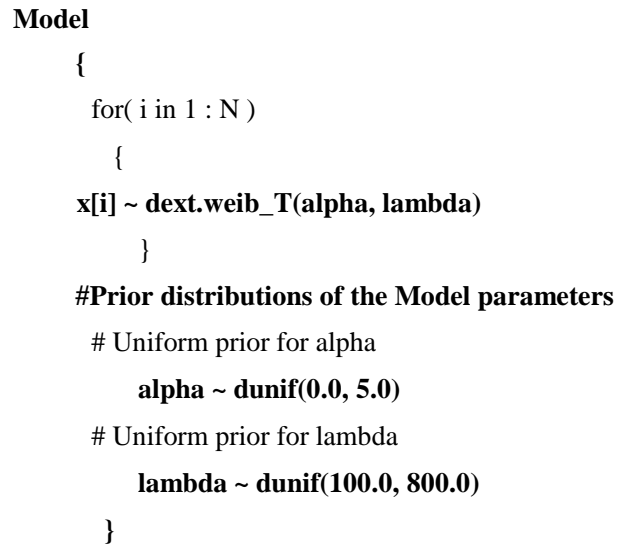

$\operatorname{list}(\mathrm{N}=86, \mathrm{x}=\mathrm{c}(4.79,7.45,10.22,15.76,26.10,28.59,35.52,41.49$, 42.66, 44.36, 45.53, 58.27, 62.96, 74.70, 81.63, 100.71, 102.06, $104.83,110.79,118.36,122.73,145.03,149.40,152.80,156.85$, 162.20, 164.97, 168.60, 173.82, 179.95, 182.72, 195.72, 203.93, 206.06, 222.26, 238.27, 241.25, 249.99, 256.17, 282.57, 282.62, 284.11, 294.45, 318.86, 323.46, 329.11, 340.30, 344.67, 353.94, 398.56, 405.70, 407.51, 422.36, 429.93, 461.47, 482.62, 491.46, $511.83,526.64,532.23,537.13,543.06,560.75,561.60,589.96$,

\author{
$592.09,610.75,615.65,630.52,673.74,687.92,698.15,753.05$, \\ 768.25, 801.06, 828.22, 849.97, 885.02, 892.27, 911.90, 951.69, \\ 962.59, 965.04, 976.98, 986.92, 1025.94)) \\ Initial values \\ \# Chain 1 \\ list $($ alpha $=0.01$, lambda $=150.0$ ) \\ \# Chain 2 \\ list $($ alpha $=2.00$, lambda $=300.0)$
}

We run the model to generate two Markov Chains at the length of 40,000 with different starting points of the parameters. The convergence is monitored using trace and ergodic mean plots, we find that the Markov Chain converge together after approximately 2000 observations. Therefore, burnin of 5000 samples is more than enough to erase the effect of starting point(initial values). Finally, samples of size 7000 are formed from the posterior by picking up equally spaced every fifth outcome, i.e. thin $=5$, starting from 5001.This is done to minimize the auto correlation among the generated deviates. Therefore, we have the posterior sample $\left\{\alpha_{1 \mathrm{i}}, \lambda_{1 \mathrm{i}}\right\}, \mathrm{i}=1, \ldots, 7000$ from chain 1 and $\left.\alpha_{2 \mathrm{i}}, \lambda_{2 \mathrm{i}}\right\}, \mathrm{i}=1, \ldots, 7000$ from chain 2 .

The chain 1 is considered for convergence diagnostics plots. The visual summary is based on posterior sample obtained from chain 2 whereas the numerical summary is presented for both the chains.

\subsection{Convergence diagnostics}

The first step in making an inference from an MCMC analysis is to ensure that an equilibrium distribution has indeed been reached by the Markov chain, i.e., that the chain has converged. For each parameter, we started the chain at an arbitrary point (the initial value or init chosen for each parameter), and because successive draws are dependent on the previous values of each parameter, the actual values chosen for the inits will be noticeable for a while. Therefore, only after a while is the chain independent of the values with which it was started. These first draws ought to be discarded as a burn-in as they are unrepresentative of the equilibrium distribution of the Markov chain.

\subsubsection{History (Trace) plot}
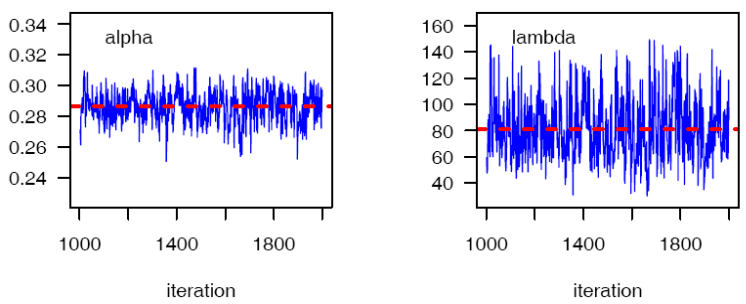

Fig 6: Sequential realization of the parameters $\alpha$ and $\lambda$.

There are several ways to check for convergence. Most methods use at least two parallel chains. Visual checks are routinely used to confirm convergence. The sequential realizations of the parameters of the model can be observed in Fig 6 .

Visual inspection of the time series plot produced by "history" suggests that the Markov chains have converged. It looks like nice oscillograms around a horizontal line without any trend. 


\subsubsection{Running Mean (Ergodic mean) Plot}

Generate a time series (iteration number) plot of the running mean for each parameter in the chain. The running mean is computed as the mean of all sampled values up to and including that at a given iteration. The convergence pattern based on ergodic averages is shown in Figure7, which indicates the convergence of the chain.
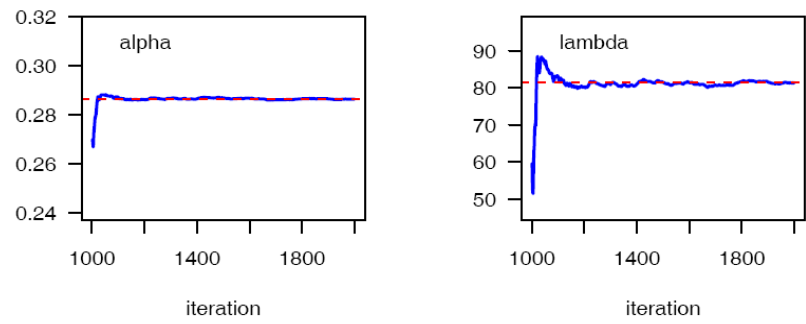

Fig 7: $\quad$ The Ergodic mean plots for alpha and lambda

\subsubsection{Autocorrelation}

The autocorrelation function is Figure8. For this model, the chains are hardly autocorrelated at all. This is good as our posterior sample contains more information about the parameters than when successive draws are correlated. The graph shows that the correlation is almost negligible. So we may consider the independent samples from the target distribution, i.e., posterior.
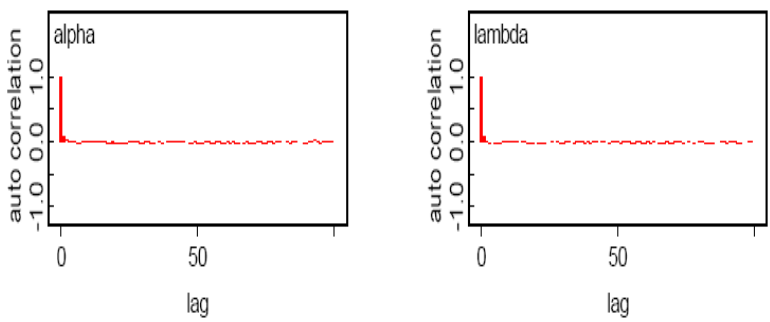

Fig 8: The autocorrelation plots for alpha and lambda

\subsubsection{Brooks-Gelman-Rubin(BGR) diagnostic}

The BGR convergence diagnostic is appropriate for the analysis of two or more parallel chains, each with different starting values which are overdispersed with respect to the target distribution.

The green line represents the between variability, the blue line represents the within variability, and the red line represents the ratio. Evidence for convergence comes from the red line being close to 1 on the y-axis and from the blue and green lines being stable (horizontal) across the width of the plot.
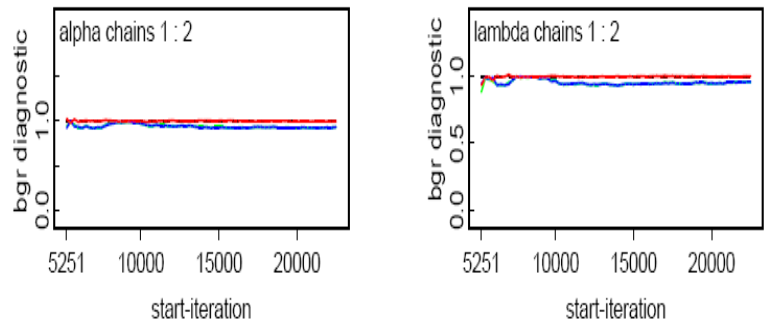

Fig 9: The BGR plots for alpha and lambda.

From the Figure9, it is clear that convergence is achieved. Thus we can obtain the posterior summary statistics.

\subsection{Numerical Summary}

In Table 2, we have considered various quantities of interest and their numerical values based on MCMC sample of posterior characteristics for MOEW model under Uniform priors. The numerical summary is based on final posterior sample (MCMC output) of 7000 samples for alpha and lambda.

$\left\{\alpha_{1 \mathrm{i}}, \lambda_{1 \mathrm{i}}\right\}, \quad \mathrm{i}=1, \ldots, 7000$ from chain 1 and $\left\{\alpha_{2 \mathrm{i},}, \lambda_{2 \mathrm{i}}\right\}$, i $=1, \ldots, 7000$ from chain 2 .

The Bayes estimates under squared error, absolute and zero-one loss functions are posterior mean, median and mode respectively as shown in Table 2. Five point summary is also given. The Highest probability density (HPD) intervals are computed the algorithm described by [1] and [2] under the assumption of unimodal marginal posterior distribution

Table 2 Numerical summaries based on MCMC sample of posterior characteristic

\begin{tabular}{|l|c|c|c|c|}
\hline \multirow{2}{*}{ Characteristics } & \multicolumn{2}{c|}{ Chain 1 } & \multicolumn{2}{c|}{ Chain 2 } \\
\cline { 2 - 5 } & alpha & lambda & alpha & lambda \\
\hline Mean & 0.3066929 & 348.6778 & 0.306648 & 346.1522 \\
\hline Standard Deviation & 0.009848204 & 132.9335 & 0.009800143 & 130.9895 \\
\hline Monte Carlo(MC) error & $1.61 \mathrm{E}-4$ & 2.076 & $1.577 \mathrm{E}-4$ & 2.3 \\
\hline Minimum & 0.2670 & 103.4000 & 0.2708 & 101.400 \\
\hline 2.5 th Percentile( $\left.\mathrm{P}_{2.5}\right)$ & 0.2871 & 154.4975 & 0.2867 & 153.395 \\
\hline First Quartile $\left(Q_{1}\right)$ & 0.3000 & 250.5000 & 0.3001 & 248.400 \\
\hline Median & 0.3070 & 324.2500 & 0.3069 & 324.100 \\
\hline Third Quartile $\left(Q_{3}\right)$ & 0.3136 & 424.4000 & 0.3135 & 419.600 \\
\hline 97.5th Percentile(P97.5) & 0.3251 & 674.5050 & 0.3248 & 665.610 \\
\hline Maximum & 0.3366 & 797.3000 & 0.3365 & 798.000 \\
\hline Mode & 0.3066484 & 292.0106 & 0.3067933 & 272.0793 \\
\hline 95\% Credible Interval & $(0.2871,0.3251)$ & $(154.49,674.50)$ & $(0.2867,0.3248)$ & $(153.395,665.61)$ \\
\hline 95\% HPD Credible Interval & $(0.2877,0.3254)$ & $(123.2,619.5)$ & $(0.2878,0.3257)$ & $(132.8,615.4)$ \\
\hline
\end{tabular}




\subsection{Visual summary}

There are two methods are used for visual summary

\subsubsection{Box plots}

The boxes represent inter-quartile ranges and the solid black line at the (approximate) centre of each box is the mean; the arms of each box extend to cover the central 95 per cent of the distribution - their ends correspond, therefore, to the $2.5 \%$ and $97.5 \%$ quantiles. (Note that this representation differs somewhat from the traditional.)
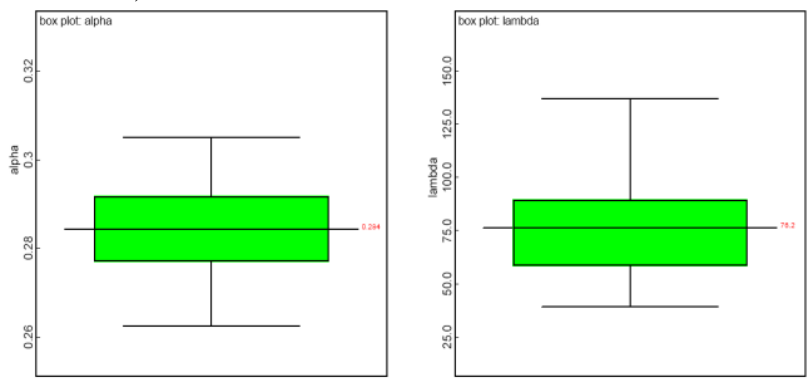

Fig 10: The boxplots for alpha and lambda.

\subsubsection{Kernel density estimates}

Histograms can provide insights on symmetric, behavior in the tails, presence of multi-modal behavior, and data outliers; histograms can be compared to the fundamental shapes associated with standard analytic distributions.

Figure 11 provides the kernel density estimate of $\alpha$ and $\lambda$. It can be seen that $\alpha$ is symmetric whereas $\lambda$ shows positive skewness.
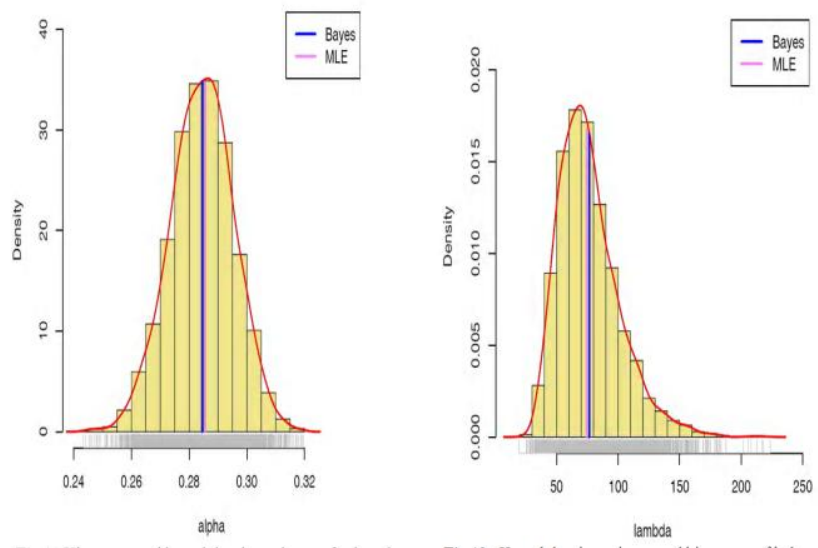

Fig 11: Histogram and kernel density estimate of $\alpha$ and $\lambda$ based on MCMC samples, vertical lines indicate the corresponding $\mathrm{ML}$ and Bays estimates.

\section{COMPARISON WITH MLE}

For the comparison with MLE we have plotted three graphs. In Figure12 the density functions $\mathrm{f}(\mathrm{x} ; \hat{\alpha}, \hat{\lambda}) \mathrm{using}$ MLEs and Bayesian estimates, computed via MCMC samples under uniform priors are plotted.

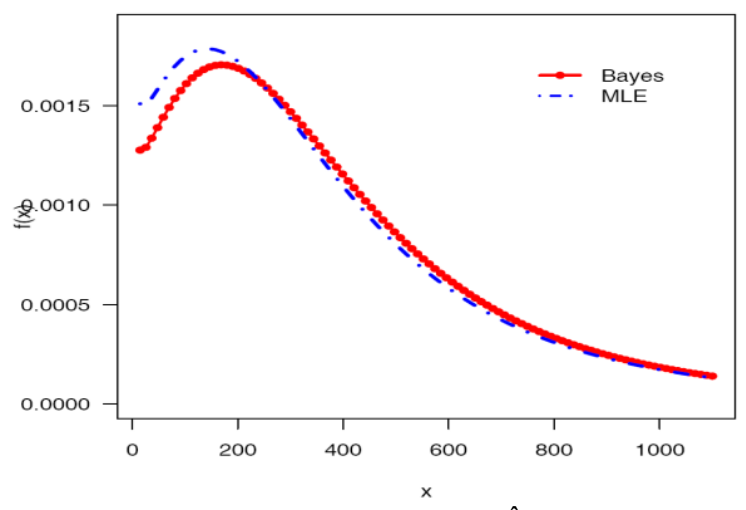

Fig 12: The density functions $\mathrm{f}(\mathrm{x} ; \hat{\alpha}, \hat{\lambda})$ using MLEs and

\section{Bayesian estimates}

The Figure13 represents the Quantile-Quantile(Q-Q) plot of empirical quantiles and theoretical quantiles computed from MLE and Bayes estimates.

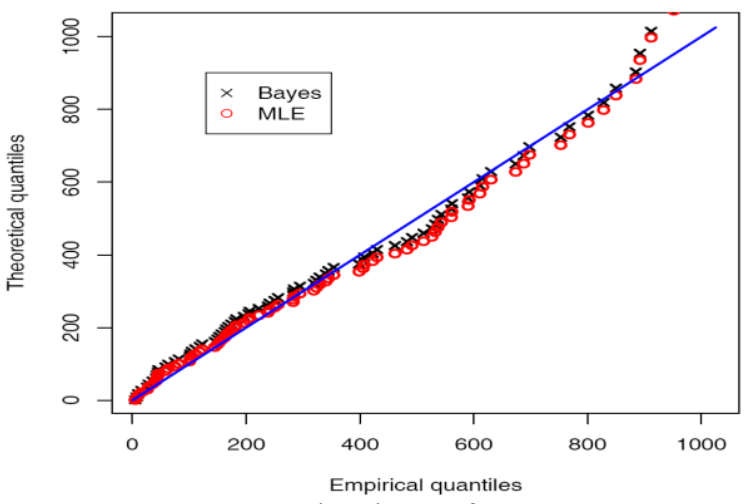

Fig 13: Quantile-Quantile(Q-Q) plot of empirical quantiles and theoretical quantiles

The Figure14 exhibits the estimated reliability function(dashed line) using Bayes estimate under uniform priors and the empirical reliability function(solid line).

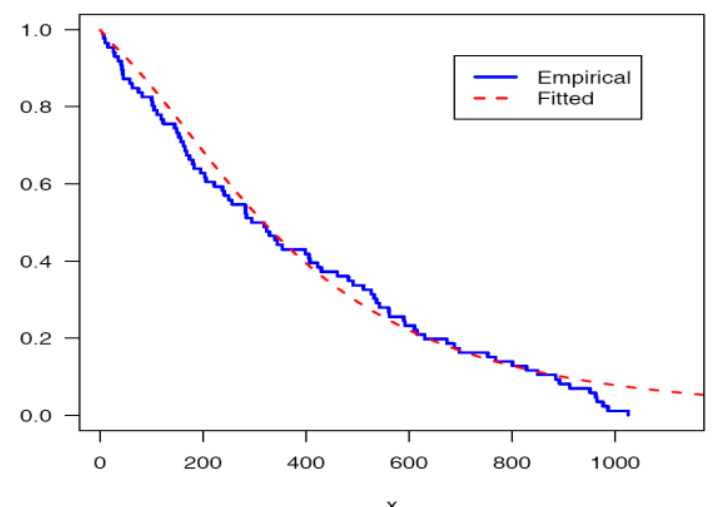

Fig 14: The estimated reliability function(dashed line) using Bayes estimate and the empirical reliability function (solid line).

It is clear from the above Figures 12, 13 and 14, the MLEs and the Bayes estimates with respect to the uniform priors are quite close and fit the data very well. 


\section{CONCLUSION}

In this paper, the statistical characteristics of the MOEW model are studied. The developed methodology for MLE and Bayesian estimation has been demonstrated on a real software reliability data set when both the parameters of the MOEW model are unknown under non-informative priors. The Bayes estimates have been obtained under various loss functions. The symmetric Bayesian credible intervals and HPD intervals have been constructed. Through the use of graphical representations, the intent is that one can gain a perspective of various meanings and associated interpretations.

The MCMC method provides an alternative method for parameter estimation of the MOEW model. It is more flexible as compared to the traditional methods such as MLE method. Moreover, 'exact' probability intervals are available rather than relying on estimates of the asymptotic variances. Indeed, the MCMC sample may be used to completely summarize posterior distribution about the parameters, through a kernel estimate. This is also true for any function of the parameters such as hazard function, mean time to failure etc. The MCMC procedure can easily be applied to complex Bayesian modeling relating to MOEW model.

We have shown that the MOEW model is suitable for the software reliability data and the tools developed for analysis can also be used for any other type of data sets.

\section{ACKNOWLEDGEMENT}

The authors would like to thank Dr. Andrew Thomas, St. Andrew's University, UK, editor and referees for several helpful comments which had improved the earlier versions of the manuscript.

\section{REFERENCES}

[1] Chen, M. H. and Shao, Q. M. (1999). Monte Carlo estimation of Bayesian credible intervals and HPD intervals, Journal of Computational and Graphical Statistics. 8(1).

[2] Chen, M., Shao, Q. and Ibrahim, J.G. (2000). Monte Carlo Methods in Bayesian Computation, Springer, NewYork.

[3] Cid, J. E. R. and Achcar, J. A., (1999). Bayesian inference for nonhomogeneous Poisson processes in software reliability models assuming nonmonotonic intensity functions, Computational Statistics \& Data Analysis 32:147-159.

[4] Hornik, K., (2004). The R FAQ (on-line). Available at http://www.ci.tuwien. ac.at/ hornik/R/.

[5] Ihaka, R.; Gentleman, R.R. (1996). R: A language for data analysis and graphics, Journal of Computational and Graphical Statistics, 5, 299-314.

[6] Jalote, P. (1991). An Integrated Approach to Software Engineering. Springer - Verlag, New York.

[7] Jiang, R. and Murthy,D.N.P. (1999). Exponentiated Weibull family: A graphical approach, IEEE Transactions on Reliability, 48(1), 68-72.

[8] Kumar, V. and Ligges, U. (2011). reliaR : A package for some probability distributions. http://cran.rproject.org/web/packages/reliaR/index.html.
[9] Lawless, J. F., (2003). Statistical Models and Methods for Lifetime Data, $2^{\text {nd }}$ ed., John Wiley and Sons, New York.

[10] Lyu M.R., (1996). Handbook of Software Reliability Engineering, IEEE Computer Society Press, McGraw Hill, 1996.

[11] Marshall, A.W., Olkin, I. (1997). A new method for adding a parameter to a family of distributions with application to the Weibull and Weibull families. Biometrika, 84(3): 641652.

[12] Marshall, A. W., Olkin, I. (2007). Life Distributions: Structure of Nonparametric, Semiparametric and Parametric Families. Springer, New York.

[13] Mudholkar, G.S. and Srivastava, D.K. (1993). Exponentiated Weibull family for analyzing bathtub failurerate data, IEEE Transactions on Reliability, 42(2), 299302.

[14] Mudholkar, G.S., Srivastava, D.K., and Freimer, M. (1995). The exponentiated Weibull family-a reanalysis of the busmotor-failure data, Technometrics, 37(4), 436-445.

[15] Nassar, M.M., and Eissa, F. H. (2003). On the Exponentiated Weibull Distribution, Communications in Statistics - Theory and Methods, 32(7), $1317-1336$.

[16] R Development Core Team (2008). R: A language and environment for statistical computing. R Foundation for Statistical Computing, Vienna, Austria. ISBN 3-900051-070, URL http://www.R-project.org.

[17] Singpurwalla, N.D. and S. Wilson (1994). Software Reliability Modeling. International Statistic. Rev., 62 3:289317.

[18] Srivastava, A.K. and Kumar V. (2011). Analysis of Software Reliability Data using Exponential Power Model. International Journal of Advanced Computer Science and Applications, Vol. 2, No. 2, February 2011, 38-45.

[19] Thomas, A. (2007). OpenBUGS, URL http://mathstat.helsinki.fi/openbugs/.

[20] Thomas, A. (2010). OpenBUGS Developer Manual, Version 3.0.2. URL http://mathstat.helsinki.fi/openbugs/

[21] Zhang T,Xie M (2007) Failure data analysis with extended Weibull distribution. Commun Statist Simul Comp, 36:579-592.

[22] Tang,Y., Xie,M., Goh,T.N.(2003).Statistical analysis of a Weibull extension model. Communications in Statistics:Theory \& Methods 32(5):913 -928.

[23] Wang,R.H., Fei,H.F.(2003).Uniqueness of the maximum likelihood estimate of the Weibull distribution tampered failure rate model. Communications in Statistics:Theory \& Methods 32:2321-2338.

[24] Xie,M., Tang,Y., Goh,T.N.(2002).A modified Weibull extension with bathtub-shaped failure rate function. Reliability Engineering System Safety 76(3):279 -285.

[25] Xie,M., Goh,T.N., Tang,Y.(2004).On changing points of mean residual life and failure rate function for some generalized Weibull distributions.Reliability Engineering \&System Safety 84:293 -299. 


\section{AUTHOR PROFILE}

ASHWINI K. SRIVASTAVA received his M.Sc in Mathematics from D.D.U.Gorakhpur University, MCA(Hons.) from U.P.Technical University and M. Phil in Computer Science from Allagappa University and submitted thesis for the award of Ph.D. in Computer Science. Currently working as Assistant Professor in Department of Computer Application in Shivharsh Kisan P.G. College, Basti, U.P. His main research interests are Software Reliability, Artificial Neural Networks, Bayesian Statistics and Data Warehousing.
VIJAY KUMAR received his M.Sc and Ph.D. in Statistics from D.D.U.Gorakhpur University. Currently working as Reader(Associate Professor) in Department of Mathematics and Statistics in DDU Gorakhpur University, Gorakhpur U.P. He has got 16 years of teaching/research experience. His main research interests are Reliability statistics, reliability models and computational statistics using OpenBUGS and R. 